\title{
Exact Density Functionals in One Dimension
}

\author{
Joachim Buschle, Philipp Maass, and Wolfgang Dieterich \\ Fakultät für Physik, Universität Konstanz, D-78457 Konstanz, Germany
}

(16 November 1999)

\begin{abstract}
We propose a new and general method for deriving exact density functionals in one dimension for lattice gases with finite-range pairwise interactions. Corresponding continuum functionals are derived by applying a proper limiting procedure. The method is based on a generalised Markov property, which allows us to set up a rather transparent scheme that covers all previously known exact functionals for one-dimensional lattice gas or fluid systems. Implications for a systematic construction of approximate density functionals in higher dimensions are pointed out.
\end{abstract}

Density functional theory provides one of the most powerful methods for studying thermodynamic properties of inhomogeneous fluids [1 1 . Various schemes are known to approximate the excess part of free energy functionals, which contains the contribution from the interactions between the molecules. Exact density functionals, however, have only been derived in one dimension $d=1$. These functionals have their merits in establishing "boundary conditions" in the sense that approximate functionals used in $d>1$ should become exact upon dimensional reduction to $d=1$. In this Letter we will present a simple general method how to derive exact density functional in one dimension for systems of particles interacting via a finite-range pair potential. In particular we will show that density functionals calculated previously [5 14] can be recovered. We will first consider lattice systems from which the corresponding continuum functionals can be straightforwardly derived by a proper limiting procedure.

The lattice Hamiltonian reads

$$
\mathcal{H}=\sum_{s} x_{s} \tilde{u}_{s}+\sum_{i<j} v_{i, j} x_{i} x_{j},
$$

where $v_{i, j}$ is the interaction potential, $\tilde{u}_{s}=u_{s}-\mu$ is the external potential minus the chemical potential $\mu$, and $x_{s}$ are the occupation numbers $\left(x_{s}=1\right.$ if site $s$ is occupied and $x_{s}=0$ else). A particularly simple situation arises for a system composed of hard rods of length $2 m$ that is described by an interaction potential $v_{i, j}=\infty$ for $j-i<2 m$ and $v_{i, j}=0$ else. For this model an exact density functional was derived by Robledo and Varea [5] (see also [6]). The corresponding continuum hard rod fluid has been treated by Percus, who succeeded to set up the first exact functional for this many-particle system with athermal interactions [7]. Later exact functionals have been derived for the multicomponent hard-rod [8] and sticky core fluid [9], as well as for the Takahashi model [10]. The perhaps most general functional was set up for a lattice system as in (1) with an arbitrary interaction potential exhibiting a finite range 11] $\left(v_{i, j}=0\right.$ for $\left.j-i \geq l\right)$. This system, that we will refer to as the "finite-range model" in the following, includes as a special case the Ising model for which various exact functionals have been derived separately for periodic boundary conditions 1214 .

We will focus here on the finite-range model with fixed boundary conditions, that means we consider a finite lattice of $M$ sites $(s=1, \ldots, M)$ and fix the occupation numbers at the boundary points $s=0$ and $s=M+1$ to be $x_{0}=x_{M+1}=1$. Our basic idea for deriving the density functional is to generate the joint probability distribution $\chi\left[x_{1}, \ldots, x_{M}\right]$ by a generalised Markov process, and then to equate the resulting expression with the Boltzmann formula $\chi\left[x_{1}, \ldots, x_{M}\right]=\mathcal{Z}^{-1} \exp (-\mathcal{H})$ (for convenient notation we set $k_{\mathrm{B}} T=1$ ).

Let us start by expressing the joint probability distribution in terms of the conditional probabilities $\omega_{s}\left[x_{s} \mid x_{s-1}, \ldots, x_{1}\right]$

$$
\chi\left[x_{1}, \ldots, x_{M}\right]=\prod_{s=1}^{M} \omega_{s}\left[x_{s} \mid x_{s-1}, \ldots, x_{1}\right] .
$$

Due to the finite range of the interaction, the conditional probabilities fulfil the Markov property $\omega_{s}\left[x_{s} \mid x_{s-1}, \ldots, x_{1}\right]=$ $\omega_{s}\left[x_{s} \mid x_{s-1}, \ldots, x_{s-(l-1)}\right]$ ( [15]; we set $x_{s}=0$ for $s<0$ for convenient notation). Factorising $\omega_{s}\left[x_{s} \mid x_{s-1}, \ldots, x_{s-(l-1)}\right]$ into $2^{l}$ terms corresponding to the possible configurations of $\left(x_{s}, \ldots, x_{s-(l-1)}\right)$ yields

$$
\omega_{s}\left[x_{s} \mid x_{s-1}, \ldots, x_{s-(l-1)}\right]=\prod_{r=0}^{l-1} \prod_{\mathbf{j} \in \mathcal{A}_{s, r}}\left[\left(\frac{a_{s}^{\mathbf{j}}}{b_{s}^{\mathbf{j}}}\right)^{x_{s}}\left(1-\frac{a_{s}^{\mathbf{j}}}{b_{s}^{\mathbf{j}}}\right)^{1-x_{s}}\right] \prod_{k=1}^{r} x_{j_{k}} \prod_{i \in I_{s, r}^{\mathbf{j}}}\left(1-x_{i}\right)
$$




$$
a_{s}^{\mathbf{j}} \equiv \mathbb{E}\left[x_{s} \prod_{k=1}^{r} x_{j_{k}} \prod_{i \in I_{s, r}^{\mathbf{j}}}\left(1-x_{i}\right)\right], \quad b_{s}^{\mathbf{j}} \equiv \mathbb{E}\left[\prod_{k=1}^{r} x_{j_{k}} \prod_{i \in I_{s, r}^{\mathbf{j}}}\left(1-x_{i}\right)\right] .
$$

$\mathbb{E}[\ldots]$ denotes an expectation value with respect to $\chi$ and $\mathcal{A}_{s, r}$ is the set of all configurations $\left(x_{s-(l-1)}, \ldots, x_{s-1}\right)$ with exactly $r$ sites being occupied. The superscript $\mathbf{j}$ specifies that particular element of $\mathcal{A}_{s, r}$, for which the $r$ sites $j_{1}, \ldots, j_{r}$ are occupied, and $I_{s, r}^{\mathbf{j}}$ is the corresponding set of all the remaining vacant sites. Note that $a_{s}^{\mathbf{j}} / b_{s}^{\mathbf{j}}$ is the probability that the site $s$ is occupied under the condition that the $r$ sites $j_{1}, \ldots, j_{r}$ are occupied while the remaining sites $i \in I_{s, r}^{\mathbf{j}}$ are empty. Although eq. (3) looks complicated, its structure becomes immediately obvious when considering the special case $l=2$, where $\omega_{s}\left[x_{s} \mid x_{s-1}\right]=\omega_{s}[1 \mid 1]^{x_{s} x_{s-1}} \omega_{s}[1 \mid 0]^{x_{s}\left(1-x_{s-1}\right)} \omega_{s}[0 \mid 1]^{\left(1-x_{s}\right) x_{s-1}} \omega_{s}[0 \mid 0]^{\left(1-x_{s}\right)\left(1-x_{s-1}\right)}$ with $\omega_{s}[1 \mid 1]=$ $\mathbb{E}\left[x_{s} x_{s-1}\right] / \mathbb{E}\left[x_{s-1}\right]$ etc.

A grand canonical functional $\Omega \equiv-\log \mathcal{Z}$ is now derived by inserting eq. (3) into eq. (2), and by using for $\chi$ the Boltzmann formula. After taking the logarithm and the expectation value on both sides of eq. (2), we obtain

$$
\Omega=\sum_{s=1}^{M} p_{s} \tilde{u}_{s}+\sum_{0 \leq i<j \leq M+1} v_{i, j} p_{i, j}+\sum_{s=1}^{M} \sum_{r=0}^{l-1} \sum_{\mathbf{j} \in \mathcal{A}_{s, r}}\left[a_{s}^{\mathbf{j}} \log \left(\frac{a_{s}^{\mathbf{j}}}{b_{s}^{\mathbf{j}}}\right)+\left(b_{s}^{\mathbf{j}}-a_{s}^{\mathbf{j}}\right) \log \left(1-\frac{a_{s}^{\mathbf{j}}}{b_{s}^{\mathbf{j}}}\right)\right]
$$

where for $r \geq 1$ and $k_{1}<\ldots<k_{r}$ we define $p_{k_{1}, \ldots, k_{r}} \equiv \mathbb{E}\left[x_{k_{1}} \ldots x_{k_{r}}\right]$. According to the definitions of $a_{s}^{\mathbf{j}}$ and $b_{s}^{\mathbf{j}}$ (see eq. (3i), $\Omega$ is a functional of all moments $p_{k_{1}, \ldots, k_{r}}$ (with $k_{r}-k_{1}<l$ ), that means it can be considered as a functional of (local) correlations up to order $l-1$. In order to derive a density functional that depends only on the occupation probabilities $p_{s}$, the correlations have to be expressed in terms of the $p_{s}$. According to the theorem of Mermin [16], this can be done in a manner that the corresponding "correlator equations" are invariant with respect to $\tilde{u}_{s}$ and we will show next that there exists a general method to achieve this [17].

To illustrate the method, we choose the special interaction potential

$$
v_{i, j}=\left\{\begin{array}{cl}
\infty & 0, \leq j-i<2 m, \\
\hat{v}_{i, j}, & 2 m \leq j-i \leq l-1<4 m .
\end{array}\right.
$$

This potential describes hard rods with lengths $2 m$, which interact via the potential $\hat{v}_{i, j}$, and it corresponds to a lattice version of the continuum Takahashi model (for $\hat{v}_{i, j}=v_{j-i}$, 18]). The special form of $v_{i, j}$ implies that in an interval of size $l-1$ there can at most two occupation numbers be equal to one. Accordingly, $a_{k}^{\mathbf{j}}$ and $b_{k}^{\mathbf{j}}$ depend only on the occupation probabilities $\left\{p_{s}\right\}$ and the two-point correlations $\left\{p_{r, s}\right\}$.

In order to calculate the two-point correlations, we consider the following three cases: (i) all $x_{s}(s=1, \ldots, M)$ are zero, (ii) exactly one of the $x_{s}$ is equal to one (and the rest equal to zero), and (iii) exactly two occupation numbers $x_{i}, x_{j}$ with $2 m \leq j-i<l$ are equal to one. By expressing the probabilities for these cases in terms of the Boltzmann formula, we find (i) $\chi\left[\right.$ all $\left.x_{s}=0\right] \equiv \chi[\mathbf{0}]=\mathcal{Z}^{-1}$, (ii) $\chi\left[x_{s}=1\right.$; rest 0$] \equiv \chi\left[x_{s}=1 ; \mathbf{0}\right]=\mathcal{Z}^{-1} \exp \left(-\tilde{u}_{s}\right.$ ), and (iii) $\chi\left[x_{i}=1, x_{j}=1 ; \mathbf{0}\right]=\mathcal{Z}^{-1} \exp \left[-\tilde{u}_{i}-\tilde{u}_{j}-\hat{v}_{i, j}\right]$ [19]. On the other hand, by considering eqs. (2,3) for the cases (i)-(iii) we obtain expressions containing the sets $\left\{p_{s}\right\}$ and $\left\{p_{i, j}\right\}$. For example, $\chi[\mathbf{0}]=\prod_{s=1}^{M}\left(1-a_{s}^{(0)} / b_{s}^{(0)}\right)$. The correlator equations then follow by multiplying the expressions for the cases (i)-(iii) in such a way that $\mathcal{Z}$ and $\left\{\tilde{u}_{s}\right\}$ cancel, i.e. in the present situation we get $\chi[\mathbf{0}] \chi\left[x_{i}=1, x_{j}=1 ; \mathbf{0}\right]=\exp \left(-\hat{v}_{i, j}\right) \chi\left[x_{i}=1 ; \mathbf{0}\right] \chi\left[x_{j}=1 ; \mathbf{0}\right]$. Inserting the $\chi^{\prime}$ s from eqs. (20.3) gives, for $i+2 m \leq j \leq i+l-1$,

$$
\begin{aligned}
& \frac{a_{j}^{(i)}}{b_{j}^{(i)}} \prod_{k=j}^{i+l-1}\left(1-\frac{a_{k}^{(0)}}{b_{k}^{(0)}}\right)=\exp \left(-\hat{v}_{i, j}\right) \frac{a_{j}^{(0)}}{b_{j}^{(0)}} \prod_{k=j}^{i+l-1}\left(1-\frac{a_{k}^{(i)}}{b_{k}^{(i)}}\right), \\
& a_{j}^{(0)} \equiv \mathbb{E}\left[x_{j} \prod_{k=j-(l-1)}^{j-1}\left(1-x_{k}\right)\right]=p_{j}-\sum_{k=j-(l-1)}^{j-2 m} p_{j, k}, \quad a_{j}^{(i)} \equiv p_{i, j}, \\
& b_{j}^{(0)} \equiv \mathbb{E}\left[\prod_{k=j-(l-1)}^{j-1}\left(1-x_{k}\right)\right]=1-\sum_{k=j-(l-1)}^{j-1} p_{k}+\sum_{k=j-(l-1)}^{j-1-2 m} \sum_{r=k+2 m}^{j-1} p_{k, r}, \quad b_{j}^{(i)} \equiv p_{i}-\sum_{k=i+2 m}^{j-1} p_{i, k} .
\end{aligned}
$$

Note that the method for determining the two-point correlator equations can also be applied for deriving higher order correlator equations. These equations would be needed for more general interaction potentials.

Having derived the correlator equations, we can view the moments in the functional $\Omega$ (雨) as functions of the $p_{s}$ and minimise $\Omega$ with respect to the $p_{s}$ to obtain the "structure equations". In fact, these equations can be derived more directly by using the relation $\chi[\mathbf{0}] \exp \left(-\tilde{u}_{i}\right)=\chi\left[x_{i}=1 ; \mathbf{0}\right]$, which gives [19] 


$$
\exp \left(-\tilde{u}_{i}\right) \prod_{s=i}^{i+l-1}\left(1-\frac{a_{s}^{(0)}}{b_{s}^{(0)}}\right)=\frac{a_{i}^{(0)}}{b_{i}^{(0)}} \prod_{s=i+2 m}^{i+l-1}\left(1-\frac{a_{s}^{(i)}}{b_{s}^{(i)}}\right)
$$

(Equation (7) together with the correlator equations (6) determines the occupation probabilities $p_{s}$.)

An explicit solution of the correlator equations (6) can be obtained for $l=2 m+1$, which means that two rods only interact if they are in contact (we set $\hat{v}_{i} \equiv \hat{v}_{i, i+2 m}$ then). The solution is

$$
p_{i, i+2 m}=\frac{e^{\hat{v}_{i}}\left(1-\sum_{k=i}^{i+2 m} p_{k}\right)+p_{i}+p_{i+2 m}}{2\left[e^{\hat{v}_{i}}-1\right]}\left[\left(1+\frac{4\left[e^{\hat{v}_{i}}-1\right] p_{i} p_{i+2 m}}{e^{\hat{v}_{i}}\left(1-\sum_{k=i}^{i+2 m} p_{k}\right)+p_{i}+p_{i+2 m}}\right)^{\frac{1}{2}}-1\right]
$$

After inserting (8) into (7) one also obtains the structure equations in explicit form. For $m=1 / 2$ and $\hat{v}_{i}=-J_{i}$ in particular, the system is equivalent to the Ising model and the structure equations simplify to the ones previously derived 20 22 by using very different approaches.

Unfortunately, for $l>(2 m+1)$ we are not able to give an explicit solution of the correlator equations (6), since then the correlator equations represent nonlinear coupled difference equations rather than a system of simple algebraic equations as in (8). The solution of the difference equations are uniquely determined by the boundary conditions induced by the two walls. Thus the $p_{i, j}$ will depend on the whole set of occupation probabilities $\left\{p_{1}, \ldots, p_{M}\right\}$ in contrast to (8), where $p_{i, i+2 m}=f_{i}\left(p_{i}, \ldots, p_{i+2 m}\right)$. The best we can do for $l>(2 m+1)$ is to reduce the problem to an equation for a single-site function $h_{s} \equiv 1-a_{s}^{(0)} / b_{s}^{(0)}$, which is the probability that the site $s$ is not occupied by a rod centre under the condition that the sites $s-(l-1), \ldots, s-1$ are also not occupied by rod centres. Using $h_{s}$, one obtains the following set of equations equivalent to (6, 6 )

$$
\begin{aligned}
h_{s} & =\frac{1-\sum_{r=s-(l-1)}^{s} p_{r}+\sum_{r=s-(l-1)}^{s-2 m} \sum_{k=r+2 m}^{s} p_{r, k}}{1-\sum_{r=s-(l-1)}^{s-1} p_{r}+\sum_{r=s-(l-1)}^{s-1-2 m} \sum_{k=r+2 m}^{s-1} p_{r, k}} \\
p_{i, j} & =p_{i} \frac{\left(1-h_{j}\right) e^{-\hat{v}_{i, j}} \prod_{k=j}^{i+l-1} h_{k}^{-1}}{1+\sum_{n=i+2 m}^{i+l-1}\left(1-h_{n}\right) e^{-\hat{v}_{i, n}} \prod_{k=n}^{i+l-1} h_{k}^{-1}} \\
e^{-\tilde{u}_{i}} & =\frac{\left(1-h_{i}\right) \prod_{s=i}^{i+l-1} h_{s}^{-1}}{1+\sum_{n=i+2 m}^{i+l-1}\left(1-h_{n}\right) e^{-\hat{v}_{i, n}} \prod_{k=n}^{i+l-1} h_{k}^{-1}}
\end{aligned}
$$

Solving (11) for $h_{i}$, and inserting this solution into (10) gives the $p_{i, j}$ in terms of the $p_{s}$. Inserting this solution then into (9) yields a closed system of equations for the $p_{s}$.

At this point we like to note that it is easy to generalise the method to lattice gases with internal degrees of freedom, where the occupation numbers can assume $q$ different values $x_{s}^{\alpha}, \alpha=1, \ldots, q$ (here and in the following the Greek letters have to be understood as superscripts). To be specific let us consider the (generalised) Potts model [23], where $v_{i, j}^{\alpha \beta}=v_{j}^{\alpha \beta} \delta_{j, i+1}$. Following the procedure described above we find (with $p_{s}^{\alpha} \equiv \mathbb{E}\left[x_{s}^{\alpha}\right], p_{s-1, s}^{\alpha \beta} \equiv \mathbb{E}\left[x_{s-1}^{\beta} x_{s}^{\alpha}\right]$, $\left.p_{s} \equiv \sum_{\alpha=1}^{q} p_{s}^{\alpha}\right)$ :

$$
\begin{aligned}
\Omega=\sum_{s=1}^{M} & \left\{\sum_{\alpha=1}^{q} \tilde{u}_{s}^{\alpha} p_{s}^{\alpha}+\sum_{\alpha, \beta=1}^{q} v_{s}^{\alpha \beta} p_{s-1, s}^{\alpha \beta}\right. \\
& +\sum_{\beta=1}^{q}\left[\sum_{\alpha=1}^{q} p_{s-1, s}^{\alpha \beta} \log \frac{p_{s-1, s}^{\alpha \beta}}{p_{s-1}^{\beta}}+\left(p_{s-1}^{\beta}-\sum_{\alpha=1}^{q} p_{s-1, s}^{\alpha \beta}\right) \log \left(1-\frac{\sum_{\alpha=1}^{q} p_{s-1, s}^{\alpha \beta}}{p_{s-1}^{\beta}}\right)\right] \\
& +\sum_{\alpha=1}^{q}\left(p_{s}^{\alpha}-\sum_{\beta=1}^{q} p_{s-1, s}^{\alpha \beta}\right) \log \left(\frac{p_{s}^{\alpha}-\sum_{\beta=1}^{q} p_{s-1, s}^{\alpha \beta}}{1-p_{s-1}}\right) \\
& \left.+\left(1-p_{s-1}-p_{s}+\sum_{\alpha, \beta=1}^{q} p_{s-1, s}^{\alpha \beta}\right) \log \left(1-\frac{p_{s}-\sum_{\alpha, \beta=1}^{q} p_{s-1, s}^{\alpha \beta}}{1-p_{s-1}}\right)\right\}
\end{aligned}
$$

and for the correlator equations, 


$$
p_{s-1, s}^{\alpha \beta}=e^{-v_{s}^{\alpha \beta}} \frac{\left(p_{s}^{\alpha}-\sum_{\gamma=1}^{q} p_{s-1, s}^{\alpha \gamma}\right)\left(p_{s-1}^{\beta}-\sum_{\delta=1}^{q} p_{s-1, s}^{\delta \beta}\right)}{\left(1-p_{s-1}-p_{s}+\sum_{\gamma, \delta=1}^{q} p_{s-1, s}^{\delta \gamma}\right)} .
$$

The structure equations read

$$
e^{-\tilde{u}_{s}^{\alpha}}=\frac{\left(1-p_{s}\right)\left(p_{s}^{\alpha}-\sum_{\gamma=1}^{q} p_{s-1, s}^{\alpha \gamma}\right)\left(p_{s}^{\alpha}-\sum_{\delta=1}^{q} p_{s, s+1}^{\delta \alpha}\right)}{p_{s}^{\alpha}\left(1-p_{s-1}-p_{s}+\sum_{\gamma, \delta=1}^{q} p_{s-1, s}^{\delta \gamma}\right)\left(1-p_{s}-p_{s+1}+\sum_{\gamma, \delta=1}^{q} p_{s, s+1}^{\delta \gamma}\right)} .
$$

For $v_{s}^{\alpha \beta}=v^{\alpha \beta}$ independent of the position $s$ these equations reduce to the ones previously derived by Percus 24].

Finally, we discuss the continuum limit of eq. (4) for the pair-potential in eq. (5) and the associated eqs. (9-11). The continuous system of size $L$ is composed of hard rods of length $\sigma$ that interact via a potential $\hat{v}(x, y)(y \geq x+\sigma)$ exhibiting a finite range $\kappa<2 \sigma$. With $\rho(x)$ being the density of rod centres and $\rho(x, y)$ the two-point density, we obtain [25]

$$
\begin{aligned}
\Omega= & \int_{0}^{L} d y \rho(y) \tilde{u}(y)+\int_{0}^{L-\sigma} d y \int_{\sigma}^{\kappa} d x \hat{v}(y, y+x) \rho(y, y+x) \\
& +\int_{0}^{L} d y\left\{\left[\rho(y)-\int_{y-\kappa}^{y-\sigma} d x \rho(x, y)\right]\left[\log \left(\frac{\rho(y)-\int_{y-\kappa}^{y-\sigma} d x \rho(x, y)}{1-\int_{y-\kappa}^{y} d x \rho(x)+\int_{y-\kappa}^{y-\sigma} d x \int_{x+\sigma}^{y} d z \rho(x, z)}\right)-1\right]\right\} \\
& +\int_{0}^{L} d y \int_{y-\kappa}^{y-\sigma} d x \rho(x, y)\left[\log \left(\frac{\rho(x, y)}{\rho(x)-\int_{x+\sigma}^{y} d z \rho(x, z)}\right)-1\right] .
\end{aligned}
$$

The set of eqs. (9-11) becomes

$$
\begin{gathered}
h(y)=\frac{\rho(y)-\int_{y-\kappa}^{y-\sigma} d z \rho(z, y)}{1-\int_{y-\kappa}^{y} d z \rho(z)+\int_{y-\kappa}^{y-\sigma} d x \int_{x+\sigma}^{y} d z \rho(x, z)}, \\
\rho(x, y)=\frac{\rho(x) h(y) \exp [-\hat{v}(x, y)] \exp \left(\int_{y}^{x+\kappa} d z h(z)\right)}{1+\int_{x+\sigma}^{x+\kappa} d z h(z) \exp [-\hat{v}(x, z)] \exp \left(\int_{z}^{x+\kappa} d u h(u)\right)} \\
\exp [-\tilde{u}(y)]=\frac{h(y) \exp \left(\int_{y}^{y+\kappa} d z h(z)\right)}{1+\int_{y+\sigma}^{y+\kappa} d z h(z) \exp [-\hat{v}(x, z)] \exp \left(\int_{z}^{y+\kappa} d u h(u)\right)} .
\end{gathered}
$$

Here $h(y) d y$ is the probability that the interval $(y, y+d y)$ is occupied by a rod centre under the condition that there is no rod centre in the interval $(y-\kappa, y)$. An explicit solution for $h(y)$ and hence for $\rho(x, y)$ can readily be obtained for the sticky-core fluid [26], and inserting this solution into (15) yields the functional derived in [9].

The functional in eq. (15) contains both the density and the density correlations, and to express the density correlations in terms of the density $\rho(x)$ one has to solve a nonlinear integral equation for $h(x)$. This form of the functional reflects the ad-hoc type of ansatz used in the weighted density functional formalism [27], which turned out to be one of the most successful methods in the theory of inhomogeneous fluids. The smoothing of the density on a local scale by a "weighting function" is in particular important to describe more subtle properties, as, for example, the wetting behaviour in the presence of confining walls [28].

It would be desirable to establish a systematic procedure for deriving density functionals in higher dimensions $d>1$. The method presented in this work can straightforwardly be generalised to higher dimensions as will be shown in forthcoming work. To keep the problem tractable, some approximations are needed in $d>1$, but the resulting functionals will by construction satisfy the requirement of becoming exact upon dimensional reduction to $d=1$. In comparison to the methods applied in previous work 11], the procedure based on the Markov chain has the advantage that the functionals are obtained directly and no functional integration of the structure equations is needed.

We gratefully acknowledge financial support from the Deutsche Forschungsgemeinschaft (SFB 513 and Ma 1636/21). 
[1] D. W. Oxtoby, in Liquids, Freezing and the Glass Transition, Vol. LI, Les Houches Summer School Lectures, edited by J. P. Hansen, D. Levesque, and J. Zinn-Justin, (Elsevier, Amsterdam, 1991).

[2] R. Evans, in Fundamentals of Inhomogeneous Fluids, edited by D. Henderson (Marcel Dekker, New York, 1992$)$, p. 85.

[3] J. K. Percus, Acc. Chem. Rev. 27, 8 (1994).

[4] H. Löwen, Phys. Rep. 237, 251 (1994).

[5] A. Robledo and C. Varea, J. Stat. Phys. 26, 513 (1981).

[6] J. Buschle, P. Maass, and W. Dieterich, J. Stat. Phys. 99, no.1/2, to appear in April 2000.

[7] J. K. Percus, J. Stat. Phys. 15, 505 (1976).

[8] T. K. Vanderlick, H. T. Davies, and J. K. Percus, J. Chem. Phys. 91, 7136 (1989).

[9] J. K. Percus, J. Stat. Phys. 28, 67 (1982).

[10] J. K. Percus, J. Phys. Condensed Matter 1, 2911 (1989).

[11] J. K. Percus, J. Stat. Phys. 60, 221 (1990).

[12] J. K. Percus, Phys. Rev. B 38, 11737 (1988).

[13] M. Q. Zhang and J. K. Percus, J. Stat. Phys. 56, 695 (1989).

[14] L. Šamaj and J. K. Percus, J. Stat. Phys. 73, 235 (1993).

[15] It is easy to prove the Markov property rigorously by using the explicit form of $\chi$ given by the Boltzmann formula. On the other hand, to deal with periodic boundary conditions, $x_{0}=x_{M+1}$, our method can also be used by modifying the generalised Markov property. For the one-dimensional Ising model with periodic boundary conditions, for example, the Markov property reads $\omega_{s}\left[x_{s} \mid x_{s-1}, \ldots, x_{0}\right]=\omega_{s}\left[x_{s} \mid x_{s-1}, x_{0}\right]$. Analogous generalisations are possible for periodic systems with one- 13] and multi-channel connection [14].

[16] N. D. Mermin, Phys. Rev. B 137, 1441 (1965).

[17] An alternative way to derive "correlator equations" appears to be a minimisation of $\Omega$ with respect to all $p_{k_{1}, \ldots, k_{l}}$. Indeed, for the Takahashi model, this method reproduces the exact results (see eq. (6)).

[18] H. Takahashi, Proceedings of the Physico-Mathematical Society of Japan (Nippon Suugaku-Buturigakkwai Kizi Tokyo) 24: 60 (1942). For a translation from the German see Mathematical Physics in One Dimension, ed. by E. H. Lieb and D. C. Mattis, (Academic Press, New York, 1966), p. 25.

[19] For points $i$ (or $j$ ) near the boundary, i.e. $2 m \leq i<l$ (or $M+1-a<j \leq M+1-2 m$ ) there would be an additional factor $\exp \left(-\hat{v}_{0, i}\right)\left(\right.$ or $\left.\exp \left(-\hat{v}_{j, M+1}\right)\right)$ due to the fixed rods at the wall. In the correlator equations (6) this factor cancels, while in the structure equation (耳) one has to replace $\tilde{u}_{i}$ by $\tilde{u}_{i}+\hat{v}_{0, i}\left(\right.$ or $\left.\tilde{u}_{i}+\hat{v}_{i, M+1}\right)$.

[20] J. K. Percus, J. Stat. Phys. 16, 299 (1977).

[21] C. F. Tejero, J. Stat. Phys. 48, 531 (1987).

[22] L. Šamaj, Physica A 153, 517 (1988).

[23] R. B. Potts, Proc. Camb. Phil. Soc. 48, 106 (1952); R. B. Potts and J. C. Ward, Progr. Theor. Phys. (Kyoto) 13, 38 (1955).

[24] J. K. Percus, J. Math. Phys. 23, 1162 (1982).

[25] When taking the continuum limit, the Boltzmann probability becomes a probability density. Taking this into account one obtains $\Omega_{\text {cont. }}=\Omega_{\text {discr. }}-\log (\Delta y) \sum_{s=1}^{M} p_{s}+\mathrm{o}(\Delta y) / \Delta y$, where $\Delta y$ is the discretisation length.

[26] R. J. Baxter, J. Chem. Phys. 49, 2770 (1968).

[27] W. A. Curtin and N. W. Ashcroft, Phys. Rev. B 32, 2909 (1985).

[28] S. Dietrich, in Phase Transitions and Critical Phenomena, edited by C. Domb and J. Lebowitz, Vol. 12 (Academic Press, London, 1988) p. 1. 